DOI: https://doi.org/10.46296/yc.v4i7edespdic.0078

\title{
MALTEADO Y VARIEDAD DE MAÍZ EN LAS CARACTERÍSTICAS FISICOQUÍMICAS Y SENSORIALES EN UNA BEBIDA REFRESCANTE
}

\section{MALTED AND VARIETY OF CORN IN THE PHYSICOCHEMICAL AND SENSORY CHARACTERISTICS IN A REFRESHING DRINK}

\author{
Moreira-Vera David ${ }^{1 *}$; Ormaza-Loor Gema²; Quiroz-Saltos Kevin ${ }^{3}$ \\ ${ }^{1}$ Docente Investigador Titular de la Escuela Superior Politécnica Agropecuaria de \\ Manabí "Manuel Félix López" ESPAM MFL. Calceta, Ecuador. Orcid: \\ http://orcid.org/0000-0002-7020-4178 \\ ${ }^{2}$ Estudiante egresada Carrera de Agroindustria de la Escuela Superior Politécnica \\ Agropecuaria de Manabí "Manuel Félix López" ESPAM MFL. Calceta, Ecuador. Orcid: \\ https://orcid.org/0000-0002-7203-159X \\ ${ }^{3}$ Estudiante egresado Carrera de Agroindustria de la Escuela Superior Politécnica \\ Agropecuaria de Manabí "Manuel Félix López" ESPAM MFL. Orcid: \\ https://orcid.org/0000-0003-0964-0584
}

*Correo: dmoreira@espam.edu.ec

\begin{abstract}
Resumen
El objetivo de esta investigación fue determinar los efectos del malteado y las variedades de maíz sobre las características fisicoquímicas y sensoriales en una bebida refrescante. Se aplicó un diseño completamente al azar (DCA) en arreglo bifactorial $A \times B$ siendo el factor $A$ tres variedades de maíz y el factor B la condición del maíz (malteado y sin maltear), obteniendo 6 tratamientos y se realizó tres repeticiones, resultando 18 unidades experimentales de $2000 \mathrm{ml}$ de bebida refrescante. Las variables valoradas fueron: fisicoquímicas $(\mathrm{pH}$, acidez, sólidos solubles y sólidos en suspensión) donde se detectaron diferencias significativas entre las bebidas con relación al $\mathrm{pH}$, acidez y grados Brix, obteniéndose como mejores tratamientos al $T_{1}, T_{3}$ y $T_{5}$, siendo éstas de condición malteado. En los análisis sensoriales (calidad general) realizado mediante la prueba de ordenamiento resultó como mejor tratamiento $\mathrm{T}_{5}$; En las pruebas microbiológicas realizadas a la bebida sobre Coliformes totales, Escherichia coli, mohos y levaduras reflejó ausencia.
\end{abstract}

Palabras clave: Maltear; maíz malteado, macerar; enzimas; bebida refrescante.

\begin{abstract}
The objective of this research was to determine the effects of malting and maize varieties on physicochemical and sensory characteristics in a refreshing beverage. A completely random design (DCA) was applied in bifactorial arrangement $A \times B$ being factor $A$ three varieties of maize and factor $B$ the condition of maize (maltexed and une malted), obtaining 6 treatments and performed three repetitions, resulting in 18 experimental units' of $2000 \mathrm{ml}$ of refreshing beverage. The variables assessed were physicochemical ( $\mathrm{pH}$, acidity, soluble solids, and suspended solids) where significant differences were detected between the drinks in relation to $\mathrm{pH}$, acidity, and Brix grades, obtaining as better treatments to $T_{1}, T_{3}$ and $T_{5}$, these being of matt condition. In sensory analyses (general quality) performed using the ordering test resulted as better T5 treatment; In microbiological tests performed on the drink on total coliforms, Escherichia coli, molds and yeasts reflected absence.
\end{abstract}

Keywords: Malting; malted corn; macerate; enzymes; refreshing drink.

Información del manuscrito:

Fecha de recepción: 05 de noviembre de 2020.

Fecha de aceptación: 14 de diciembre de 2020.

Fecha de publicación: 21 de diciembre de 2020. 


\section{Introducción}

El uso de ingredientes cada vez más sintéticos en la elaboración de bebidas refrescantes, es un gran problema que surge en el país, esto se debe a la gran demanda que existe de estas bebidas en el mercado debido a su bajo costo (Harding \& Lovenheim, 2014), razón que motiva a la poca innovación por parte de las industrias al no utilizar materias primas de origen natural, incrementándose la disponibilidad de bebidas elaboradas con materias primas sintéticas, como acidulantes, saborizantes, estabilizantes, colorantes y preservantes (Álvarez, 2012).

Por otro lado, al no evidenciarse información de una bebida refrescante utilizando diferentes variedades de maíz malteado y sin maltear, se desconocen las posibles diferencias y el efecto que esto provoque en las características fisicoquímicas y sensoriales en una bebida de este tipo.

Es por esta razón que se utilizó como materia prima tres variedades de maíz: criollo amarillo, blanco Pichilingue 513 y duro Dekalb 399 en una bebida refrescante, se optó por estas variedades debido a que en el caso del maíz criollo amarillo se tienen conocimientos muy artesanales sobre los productos elaborados a base de este grano. Con lo que respecta al maíz blanco y duro porque son variedades muy cultivadas dentro de la provincia de Manabí.

Es por eso por lo que se eligió el maíz como materia prima para diseñar una variedad de producto atractivo e innovador, para incrementar su consumo y presentar una nueva alternativa de producto terminado; con esta investigación se determina el efecto del malteado y las variedades de maíz sobre las características fisicoquímicas y sensoriales en una bebida refrescante.

\section{Materiales y métodos}

\subsection{Obtención del maíz malteado}

Se receptó el maíz y se procedió a separarlo manualmente de impurezas. Luego se remojaron los granos de maíz con agua purificada, en una relación en kilogramos de 1:1,5 (granos: agua) por un lapso de 12 horas a temperatura ambiente $\left(28+/-2^{\circ} \mathrm{C}\right)$. Transcurrido el periodo de remojo, los granos fueron 
llevados a germinar a temperatura ambiente $\left(28+/-2^{\circ} \mathrm{C}\right)$ durante 4 días, humectándolos con agua purificada cada 8 horas mediante proceso de aspersión, alcanzando una humedad mayor a 30\%; obteniéndose una radícula de un promedio de $4 \mathrm{~cm}$ y un talluelo. Una vez germinado los granos, estos fueron sometidos a un proceso de secado (60ํ C por 2 horas) y tostado $\left(110^{\circ} \mathrm{C}\right.$ por 2 horas), en una estufa Memmert® UFE 600. Luego, el maíz se enfrió y las radículas fueron removidas manualmente mediante frotación.

\subsection{Obtención de la bebida refrescante de maíz}

El maíz con la condición malteado o sin maltear es triturado en un molino manual. El maíz triturado (15\%) y el agua (85\%) se mezclaron entre sí en una olla de maceración doble fondo. La maceración se realizó de manera escalonada utilizando una escala de temperatura y tiempo de $45^{\circ} \mathrm{C}$ por 20 minutos, $55^{\circ} \mathrm{C}$ por 15 minutos y $65^{\circ}$ C por 40 minutos. Al finalizar el proceso de maceración el líquido es filtrado del sólido. El mosto obtenido se coció por 60 minutos, durante ese tiempo se realizó la adición del lúpulo $(0,05 \%)$. Después de esto, se adicionó el azúcar (5\%), la panela $(3 \%)$ y la esencia de vainilla $(0,35 \%)$. El líquido se filtró y se colocó la bebida en envases de plástico transparente con una capacidad de 5 litros y se llevó a una temperatura de $4^{\circ} \mathrm{C}$ para su sedimentación por un lapso de 48 horas. La bebida se envasó en botellas de vidrio ámbar previamente esterilizado de $330 \mathrm{~mL}$ de capacidad. Cada una de las bebidas embotellas fueron pasteurizadas a $85^{\circ} \mathrm{C} / 15 \mathrm{~min}$. Las bebidas fueron almacenadas en una cámara de refrigeración a $4^{\circ} \mathrm{C}$ para su conservación. Luego de 2-3 días se procedió a realizar las pruebas fisicoquímicas y sensoriales.

\subsection{Análisis estadístico}

Los datos obtenidos se expresarán en términos de media \pm desviación estándar. Los datos se analizaron mediante un análisis de la varianza ADEVA con un nivel de confianza del $95 \%$, y una prueba de diferencia de medias Tukey. Además, se empleará la prueba de Kruskall Wallis y Jonckheere-Terpstra. Los resultados obtenidos de los análisis sensoriales se analizaron utilizando el método estadístico de Friedman. El análisis de los datos se los efectuó por medio 
del programa SPSS 21 Versión Libre.

\section{Resultados y discusión}

Los supuestos de normalidad y homogeneidad indicaron que la variable $\mathrm{pH}$ cumple con estos supuestos, como se observa en la tabla 1 y 2, indicando así que los datos se distribuyen de manera normal; mientras que, para las variables acidez, ${ }^{\circ B}$ rix y sólidos en suspensión sólo se cumple con uno de los supuestos, por lo cual se procedió a realizar pruebas no paramétricas (Kruskall Wallis y Jonckheere-Terpstra).

Tabla 1. Test de Shapiro Wilk.

\begin{tabular}{|c|c|c|c|}
\hline \multirow{2}{*}{ Variables } & \multicolumn{3}{|c|}{ Prueba de normalidad } \\
\cline { 2 - 4 } & Estadístico & gl & Sig. \\
\hline $\mathrm{pH}$ & 0,826 & 3 & 0,247 \\
Acidez & 0,782 & 3 & 0,089 \\
`Brix & 0,750 & 3 & 0,000 \\
Sólidos en suspensión & 0,839 & 3 & 0,287 \\
\hline
\end{tabular}

Elaborado por: Autores (2020).

Tabla 2. Test de Levene.

\begin{tabular}{|c|c|c|c|c|}
\hline Variables & $\mathbf{F}$ & $\mathbf{g l}^{\mathbf{1}}$ & $\mathbf{g l}^{\mathbf{2}}$ & Sig. \\
\hline $\mathrm{pH}$ & 2,413 & 5 & 12 & 0,098 \\
Acidez & 4,029 & 5 & 12 & 0,022 \\
○Brix & 3,200 & 5 & 12 & 0,046 \\
Sólidos en suspensión & 4,473 & 5 & 12 & 0,016 \\
\hline
\end{tabular}

Elaborado por: Autores (2020).

\section{1. $\mathrm{pH}$}

En la tabla 3 se presenta el análisis de varianza, en donde se establecieron diferencias estadísticamente significativas $(p<0,05)$ para el factor $A$ (variedad de maíz), el factor $B$ (condición de maíz) y la interacción de estos dos factores sobre la variable $\mathrm{pH}$, esto quiere decir que tanto las variedades como la condición de maíz influyen directamente en la bebida refrescante.

Tabla 3. Resultados del análisis de varianza en la variable pH.

\begin{tabular}{|l|c|c|c|c|c|}
\hline Origen & gl & $\begin{array}{c}\text { Suma de } \\
\text { cuadrados tipo III }\end{array}$ & $\begin{array}{c}\text { Media } \\
\text { cuadrática }\end{array}$ & F & Sig. \\
\hline Total corregida & 17 & 1,518 & & & \\
\hline
\end{tabular}




\begin{tabular}{|l|c|c|c|c|c|}
\hline Modelo corregido & 5 & $1,514^{\mathrm{a}}$ &, 303 & 825,688 &, $000^{* *}$ \\
\hline Factor_A & 2 &, 065 &, 032 & 88,288 &, $000^{* *}$ \\
\hline Factor_B & 1 & 1,439 & 1,439 & 3925,470 &, $000^{* *}$ \\
\hline Factor_A * Factor_B & 2 &, 010 &, 005 & 13,197 &, $001^{* *}$ \\
\hline Error & 12 &, 004 &, 000 & & \\
\hline
\end{tabular}

${ }^{\star *}$ Altamente significativo

Elaborado por: Autores (2020).

El pH del maíz sin maltear oscila entre 5,5 y 8,0 Yao et al., (2017), mientras que para un maíz malteado, Galecio \& Haro (2012), publicaron valores de 5,9 a 6,2; pudiendo interpretar los valores de pH del maíz sin maltear entre ligeramente ácido, neutro y débilmente alcalino, mientras que para el maíz malteado el rango de $\mathrm{pH}$ es menor, siendo este interpretado como ligeramente ácido.

Razón por la cual es importante destacar que las bebidas que obtuvieron un $\mathrm{pH}$ menor son las elaboradas con maíz malteado, por el contrario, las bebidas de maíz sin maltear obtuvieron un $\mathrm{pH}$ más alto. Esto contrasta con lo mencionado por Olivares \& Ricaldi (2013), los cuales indicaron que el malteado tuvo influencia en el $\mathrm{pH}$ de una harina de maíz, ya que entre mayor fue el tiempo de germinación de maíz, menor fue el pH obtenido en la harina evaluada.
Además, señalan Mosher \& Trantham (2016) que en el proceso del malteo son generadas enzimas por la semilla en crecimiento, entre ellas la fitasa, la cual libera el fosfato de la fitina que disminuye el $\mathrm{pH}$ del grano, siendo este un posible motivo por el cual se obtuvo valores menores en las bebidas elaboradas con maíz malteado.

La Norma INEN 2304 (2017), para bebidas refrescantes establece como requisito un $\mathrm{pH}$ mínimo de 2,0 y máximo de 4,5 lo cual se puede observar en la tabla 5 que los valores de $\mathrm{pH}$ obtenidos en los diferentes tratamientos no se encuentran dentro del rango establecido en la norma. Sin embargo, mediante la figura 1 de cajas y bigotes se puede observar que estadísticamente el mejor tratamiento que presentó un $\mathrm{pH}$ menor fue el $\mathrm{T}_{3}$ (Maíz blanco Pichilingue 513 malteado). 
Tabla 4. Valores promedios de $\mathrm{pH}$ en una bebida refrescante.

\begin{tabular}{|c|c|}
\hline & Variable \\
\hline Tratamientos & pH \\
\hline & \\
\hline & \\
T2 (Maíz criollo amarillo sin maltear) & $6,2067 \pm 0,01155 \mathrm{e}$ \\
T4 (Maíz blanco Pichilingue 513 sin maltear) & $6,0667 \pm 0,00577 \mathrm{~d}$ \\
T6 (Maíz Dekalb 399 sin maltear) & $6,0133 \pm 0,01528 \mathrm{c}$ \\
T1 (Maíz criollo amarillo malteado) & $5,5867 \pm 0,02309 \mathrm{~b}$ \\
T5 (Maíz Dekalb 399 malteado) & $5,5067 \pm 0,00577 \mathrm{a}$ \\
T3 (Maíz blanco Pichilingue 513 malteado) & $5,4967 \pm 0,03512^{\mathrm{a}}$ \\
& \\
\hline Factor A & ${ }^{\star \star}$ \\
\hline Maíz Criollo Amarillo & $5,8967 \pm 0,33998 \mathrm{~b}$ \\
Maíz Blanco Pichilingue 513 & $5,7817 \pm 0,31301 \mathrm{a}$ \\
Maíz Duro Dekalb 399 & $5,7600 \pm 0,27770^{\mathrm{a}}$ \\
\hline Factor B & $6,096 \pm 0,08705 \mathrm{~b}$ \\
\hline Maíz Sin Maltear & $5,530 \pm 0,04770 \mathrm{a}$ \\
\hline Maíz Malteado &
\end{tabular}

Los valores corresponden al promedio de la variable $\mathrm{pH} \pm$ desviación estándar. a,b,c,d y e difieren entre sí por la prueba de Tukey $(p<0,05)$

${ }^{* *}$ Altamente significativo

Elaborado por: Autores (2020)

Figura 1. Gráfico de cajas y bigotes para todos los tratamientos en la variable $\mathrm{pH}$

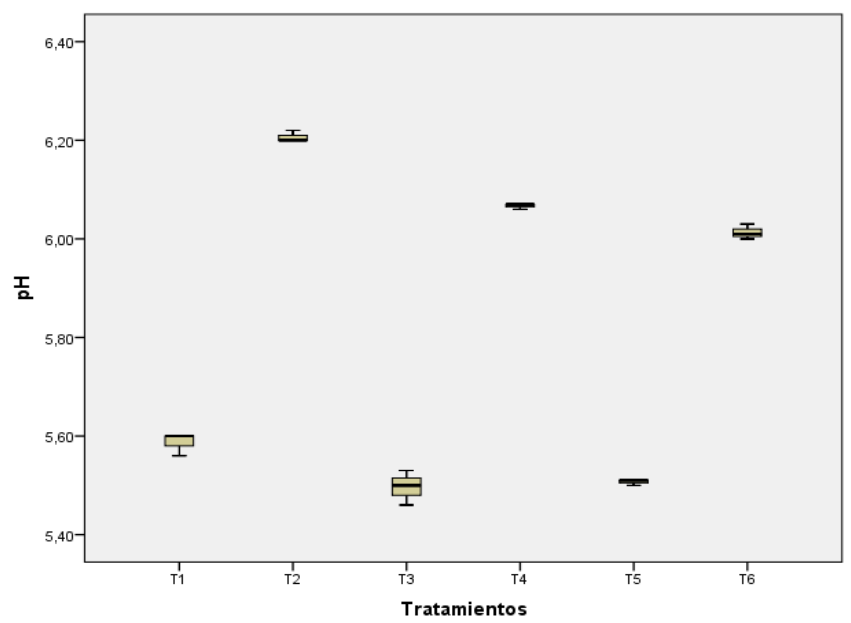

Fuente: Autores (2020)

\subsection{Acidez}

Mediante la prueba de Kruskal Wallis se estableció que el Factor A no influye en la acidez de la bebida refrescante, pero en cuanto al Factor B y en la interacción de estos dos factores si existió significancia $(p<0,05)$, esto indica que la variedad de maíz no tiene influencia, mientras que la condición del maíz si afecta significativamente sobre esta variable. 
Tabla 5. Resultados de la prueba de Kruskal Wallis para la variable acidez.

\begin{tabular}{|c|l|r|r|r|}
\hline & \multicolumn{1}{|c|}{ Estadístico } & \multicolumn{1}{|c|}{ Factor A } & \multicolumn{1}{c|}{ Factor B } & \multicolumn{1}{c|}{ Interacción } \\
\hline Acidez & Chi-cuadrado &, 878 & 13,198 & 14,133 \\
& gl. & 2 & 1 & 5 \\
& Sig. asintótica & 0,645 &, 000 & 0,015 \\
\hline
\end{tabular}

Elaborado por: Autores (2020)

Por medio de pruebas no paramétricas (Kruskall Wallis), se puede identificar diferencias significativas entre los tratamientos para esta variable, al clasificar estas diferencias se puede mostrar que los valores más bajos obtenidos de acidez se encuentran en los tratamientos $\mathrm{T}_{2}, \mathrm{~T}_{4}$ y $\mathrm{T}_{6}$, que pertenecen a las bebidas elaboradas con maíz criollo amarillo, blanco Pichillingue 513 y Dekalb 399 respectivamente, todos estos con la condición sin maltear; mientras que una acidez más elevada se reportó en las bebidas de maíz de las variedades ya mencionadas pero con la condición malteada.

Tabla 6. Valores promedios de acidez en una bebida refrescante

\begin{tabular}{|c|c|}
\hline Tratamientos & Variable \\
\cline { 2 - 2 } & Acidez \\
\hline T5(Maíz Dekalb 399 malteado) & $0,5733 \pm 0,02309 \mathrm{c}$ \\
T1 (Maíz criollo amarillo malteado) & $0,5500 \pm 0,01732 \mathrm{bc}$ \\
T3 (Maíz blanco Pichilingue 513 malteado) & $0,5400 \pm 0,07211 \mathrm{bc}$ \\
T6 (Maíz Dekalb 399 sin maltear) & $0,3967 \pm 0,04041 \mathrm{ab}$ \\
T4 (Maíz blanco Pichilingue 513 sin & $0,3267 \pm 0,04041 \mathrm{a}$ \\
maltear) & $0,3067 \pm 0,09815 \mathrm{a}$ \\
T2 (Maíz criollo amarillo sin maltear) & \\
\hline CV & 0,12 \\
\hline Kruskall Wallis & 0,015 \\
\hline
\end{tabular}

CV: Coeficiente de variación. \pm Desviación estándar $a, b$ y c difieren según Kruskall Wallis $(p<0,05)$

Elaborado por: Autores (2020)

En la investigación realizada por Casas et al., (2016), en donde se evaluó el efecto del malteado en una bebida de quinua, se pudo observar un caso similar al anterior, en donde se presentó un incremento en el porcentaje de acidez en las bebidas elaboradas con quinua malteada, estos autores mencionan que este incremento puede estar relacionado a la liberación de ácidos grasos y minerales que se producen en la etapa de germinación de los granos como consecuencia del 
desdoblamiento de nutrientes como almidón, proteína y grasa mediante la acción de enzimas; así mismo, manifiestan Mosher \& Trantham (2016), que una vez que comienza la hidratación en el proceso del malteado, el embrión y el escutelo comienzan a producir ácido.

En efecto, Faltermaier et al., (2013), expresan que, durante el malteado, especialmente en la etapa de germinación, los cambios metabólicos causan degradación de proteínas, formando moléculas más pequeñas, revelándose el aumento de todos los aminoácidos durante todas las etapas del proceso de malteado.

Con respecto a la Norma INEN 2304 (2017) para refrescos o bebidas no carbonatadas, establece un mínimo de acidez de 0,1\%, por lo que se puede evidenciar en la tabla 6 que en todos los tratamientos se cumple con lo establecido en la norma, sin embargo, mediante la figura 2 de cajas y bigotes se obtienen estadísticamente como mejores tratamiento al T5 (Maíz Dekalb 399 malteado) $T_{1}$ (Maíz criollo amarillo malteado) y $\mathrm{T}_{3}$ (Maíz blanco Pichilingue 513 malteado), los cuales obtuvieron una acidez mayor.

Figura 2. Gráfico de cajas y bigotes para todos los tratamientos en la variable acidez

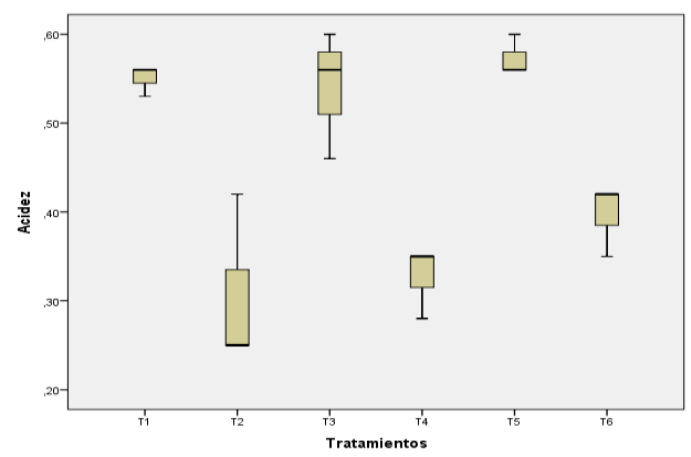

Fuente: Autores (2020)

\section{3. ${ }^{\circ}$ Brix}

Es importante destacar, que en esta variable ( ${ }^{\circ}$ Brix), ocurre lo mismo que en la variable acidez, ya que no se estableció diferencia significativa para el factor $A$, pero en cuanto al factor B y en la interacción de estos dos factores si existió significancia $(p<0,05)$, esto indica que la variedad de maíz no tiene influencia, mientras que la condición del maíz si afecta significativamente sobre esta variable.

Tabla 7. Valores promedios de ${ }^{\circ}$ Brix en una bebida refrescante

\begin{tabular}{llrrr}
\hline \multicolumn{1}{c}{ Estadístico } & \multicolumn{1}{c}{ Factor A } & Factor B & \multicolumn{1}{c}{ Interacción } \\
\hline${ }^{\circ}$ Brix & Chi-cuadrado &, 583 & 13,018 & 16,542 \\
& gl. & 2 & 1 & 5 \\
& Sig. asintótica &, 747 &, 000 &, 005 \\
\hline
\end{tabular}

Elaborado por: Autores (2020) 
Al categorizarse estas diferencias se comprobó que los ${ }^{\circ}$ Brix de las bebidas elaboradas a base de maíz sin maltear de las variedades Dekalb 399, amarillo criollo y blanco
Pichilingue $\left(T_{6}, T_{2}, T_{4}\right)$, obtuvieron valores menores de ${ }^{\circ}$ Brix, a diferencia de las bebidas elaboradas con maíz malteado de las variedades ya descritas $\left(T_{3}, T_{5}, T_{1}\right)$.

Tabla 8. Valores promedios de ${ }^{\circ}$ Brix en una bebida refrescante

\begin{tabular}{|c|c|}
\hline Tratamientos & Variable \\
\cline { 2 - 2 } & ${ }^{\circ}$ Brix \\
\hline T1(Maíz criollo amarillo malteado) & $11,333 \pm 0,0577 \mathrm{e}$ \\
T5 (Maíz Dekalb 399 malteado) & $10,500 \pm 0,0000 \mathrm{~d}$ \\
T3 (Maíz blanco Pichilingue 513 malteado) & $9,333 \pm 0,0577 \mathrm{c}$ \\
T4 (Maíz blanco Pichilingue 513 sin & $9,033 \pm 0,0577 \mathrm{~b}$ \\
maltear) & $8,433 \pm 0,0577 \mathrm{a}$ \\
T2 (Maíz criollo amarillo sin maltear) & $8,367 \pm 0,0577 \mathrm{a}$ \\
T6 (Maíz Dekalb 399 sin maltear) & \\
\hline CV & 1,11 \\
\hline Kruskall Wallis & 0,005 \\
\hline
\end{tabular}

CV: Coeficiente de variación. \pm Desviación estándar $a, b$ y c difieren según Kruskall Wallis $(p<0,05)$

Elaborado por: Autores (2020)

Este notable crecimiento del dulzor de las bebidas, según Drapala \& Hernández (2018), es producto de la hidrólisis enzimática del almidón que se da en el proceso del malteado, debido a que cereales como trigo, arroz y maíz contienen almidón en gran cantidad; el almidón se almacena en estas semillas para proporcionar energía para el crecimiento del embrión durante la germinación de las semillas. En consecuencia, señalan Mosher \& Trantham (2016), que las enzimas que se activan incluyen las que ya son producidas por la semilla y las que están esperando hidratación.
Entre las enzimas generadas por la creciente semilla se encuentra la amilasa, encargada de convertir los almidones presentes en el endospermo, en azúcares (glucosa y maltosa).

En cuanto a $\operatorname{los}^{\circ}$ Brix para bebidas 0 refrescos no carbonatados, la Norma INEN 2304 (2017), no establece un mínimo, pero si un máximo de $15^{\circ}$ Brix para este tipo de bebidas; cumpliendo todos los tratamientos con lo determinado en la norma para este parámetro, sin embargo, en la figura 3 de cajas y bigotes se presenta como mejor tratamiento 
para esta variable en estudio al $\mathrm{T}_{1}$ (Maíz criollo amarillo malteado).

Figura 3. Gráfico de cajas y bigotes para todos los tratamientos en la variable ${ }^{\circ} B r i x$.

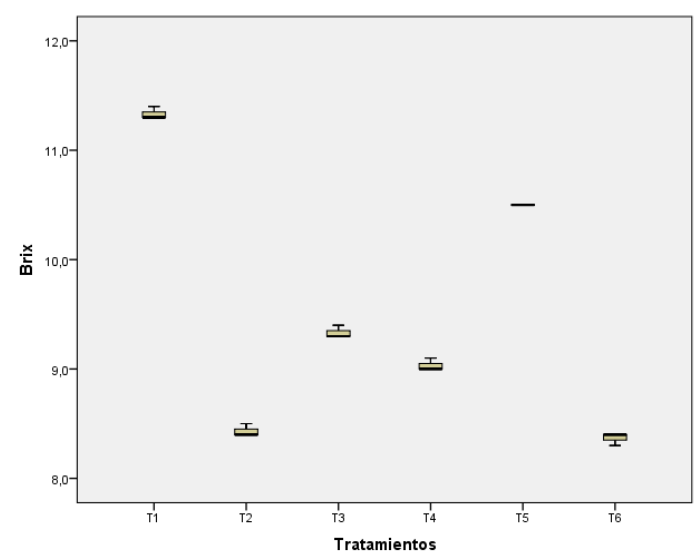

Fuente: Autores (2020)

\subsection{Sólidos en suspensión}

Se determinaron mediante la prueba de Jonckheere-Terpstra que tanto en el Factor A (Variedades de maíz), como en el Factor $B$ (condición del maíz) no existen diferencias estadísticamente significativas, de la misma manera en la interacción de estos dos factores; debido a que la significancia fue mayor que el 0,05.

Tabla 9. Prueba de Jonckheere-Terpstraa

\begin{tabular}{|l|r|}
\hline & \multicolumn{2}{|c|}{ Solidos en Suspensión } \\
\hline Número de niveles en Factor_A & 3 \\
\hline N & 18 \\
\hline Media del estadístico J-T & 54,000 \\
\hline Sig. asintót. (bilateral) &, 123 \\
\hline Número de niveles en Factor_B & 2 \\
N & 18 \\
Media del estadístico J-T & 40,500 \\
Sig. asintót. (bilateral) &, 716 \\
\hline Número de niveles en Tratamientos & 6 \\
N & 18 \\
Media del estadístico J-T & 67,500 \\
Sig. asintót. (bilateral) &, 087 \\
\hline
\end{tabular}

Elaborado por: Autores (2020)

En la tabla 9 de las medias obtenidas del porcentaje de sólidos en suspensión de las bebidas refrescantes, se refleja los resultados obtenidos en el análisis estadístico, que demuestra que todos los tratamientos son iguales con respecto a esta variable, ya que el rango varía entre 0,03 y $0,05 \%$ de sólidos en suspensión.

En la investigación publicada por De León (2016) se muestra un rango de 0,018 a $0,054 \%$ de sólidos en 
suspensión en una cerveza de maíz, este rango es semejante al reportado en las bebidas refrescantes, para lo cual mencionan los autores que esta variable es un atributo que hace que las bebidas sean claras y homogéneas, lo cual, sensorialmente lo hace atractivo al ojo humano; añade Lozano (2006) que particularmente, los jugos de frutas 0 bebidas turbias tienen sólidos de varias dimensiones distribuidos en un líquido. Por otro lado, menciona Recalde (2017), que cuando se utilizan granos en la elaboración de bebidas, se obtienen pequeñas partículas en suspensión en el producto final, por lo que recalca que en su investigación este fenómeno aumentó con la adición de una mayor concentración de granos malteados en una cerveza.

De acuerdo con lo anterior, se puede comprobar que para todos los tratamientos se utilizó la misma concentración de granos de maíz (malteados y sin maltear), por ello no existe diferencia entre los tratamientos, además de que ninguno de los factores influye en esta variable.

\subsection{Análisis sensorial}

En la evaluación sensorial que consistió en una prueba por ordenamiento, realizada a un grupo de 75 jueces no entrenados, que permitió evaluar la calidad general de las bebidas; los jueces lograron determinar el mejor tratamiento siendo este el T5 (Maíz Dekalb 399 Malteado), esto se logró identificar mediante la prueba de Friedman que otorgó la primera categoría estadística (a) como mejor tratamiento al $\mathrm{T}_{5}$ con rango promedio 2,25a. Este tratamiento presentó mayor aceptación, siendo la materia prima de condición malteada, tal como se puede evidenciar en la tabla 10, lo cual coincide con Aliaga et al., (2017) que mencionan que con la germinación se obtienen alimentos organolépticamente agradables, por lo que Kok et al., (2018), dan a conocer que durante el germinado, se liberan 0 se activan enzimas (dextrinasas, $\beta$-glucanasa, xilanasa, endo y exo-proteinasas, lipasas y dextrinasas). Estas enzimas están involucradas en la descomposición del almidón en azúcares. Por otro lado, los mismos autores también expresan que en el horneado (con el cual se detiene la germinación), 
ayuda a generar aroma, sabor y compuestos coloreados que ayudan a definir el carácter final del producto.

Tabla 9. Resultados del test de Friedman de la prueba de ordenamiento.

\begin{tabular}{|c|c|}
\hline TRATAMIENTOS & RANGO PROMEDIO \\
\hline T1(Maíz criollo amarillo malteado) & $3,43 \mathrm{~b}$ \\
T2(Maíz criollo amarillo sin maltear) & $3,65 \mathrm{~b}$ \\
T3(Maíz blanco Pichilingue 513 malteado) & $3,76 \mathrm{~b}$ \\
T4(Maíz blanco Pichilingue 513 sin maltear) & $3,73 \mathrm{~b}$ \\
T5(Maíz Dekalb 399 malteado) & $2,25 \mathrm{a}$ \\
T6(Maíz Dekalb 399 sin maltear) & $4,17 \mathrm{~b}$ \\
\hline Friedman & 450 \\
\hline Sig. & 0,03 \\
\hline
\end{tabular}

a y b difieren según Friedman $(p<0,05)$

Elaborado por: Autores (2020)

\subsection{Análisis microbiológicos}

Se detallan las pruebas microbiológicas realizadas al $\mathrm{T}_{5}$, que se identifica como mejor tratamiento desde el punto sensorial, siendo este tratamiento elaborado con maíz Dekalb 399 malteado. La bebida refrescante fue sometida a pruebas de Recuento de Coliformes totales,
Escherichia coli, y mohos y levaduras (INEN 2337, 2008).

En la tabla 11 se especifican los resultados obtenidos de las pruebas realizadas a la bebida, las mismas que reflejaron ausencia en cada uno de los parámetros medidos en dicho tratamiento, estos resultados garantizan la inocuidad del producto final.

Tabla 10. Resultados del análisis microbiológico de la bebida refrescante.

\begin{tabular}{|c|c|c|c|c|}
\hline $\begin{array}{l}\text { Identificación } \\
\text { de la muestra }\end{array}$ & $\begin{array}{l}\text { Pruebas } \\
\text { realizadas }\end{array}$ & Unidad & Resultados & Método de ensayo \\
\hline \multirow{3}{*}{$\begin{array}{c}\text { Bebida } \\
\text { refrescante de } \\
\text { maíz }\end{array}$} & $\begin{array}{c}\text { Recuento de } \\
\text { Coliformes totales } \\
\end{array}$ & $\mathrm{NMP} / \mathrm{mL}$ & Ausencia & NTE INEN 1529-6 \\
\hline & $\begin{array}{l}\text { Recuento de } \\
\text { Escherichia coli }\end{array}$ & $\mathrm{NMP} / \mathrm{mL}$ & Ausencia & NTE INEN 1529-8 \\
\hline & $\begin{array}{c}\text { Recuento de } \\
\text { Mohos y Levaduras }\end{array}$ & $\mathrm{UP} / \mathrm{mL}$ & Ausencia & $\begin{array}{c}\text { AOAC Método oficial } \\
997.02\end{array}$ \\
\hline
\end{tabular}

Elaborado por: Autores (2020) 


\section{Conclusiones}

Se identificó dentro del factor variedades de maíz que el efecto altamente significativo lo obtuvo la variable $\mathrm{pH}$, los tratamientos $\mathrm{T}_{2}$ con un valor más alto de 6,20 y el T3 con el valor más bajo 5,49; Sin embargo, no hubo incidencia en las variables acidez, `Brix y sólidos en suspensión en la bebida refrescante obtenida.

En los efectos del malteado del maíz se obtuvo diferencias altamente significativas en las variables fisicoquímicas de $\mathrm{pH}$, acidez y ${ }^{\circ}$ Brix; mientras que en las variables sólidos en suspensión no hubo efectos significativos.

En el análisis sensorial se estableció como mejor tratamiento al $\mathrm{T}_{5}$, que corresponde a la bebida elaborada con maíz de la variedad Dekalb 399 y la condición malteado.

\section{Bibliografía}

Aliaga, S., Mamani, F., \& Mamani, N. (2017). Potencial alimenticio de los germinados de Cañahua (Chenopodium pallidicaule Aellen). APTHAPI, 3.

Álvarez, Y. (2012). Elaboración y caracterizacion de dos bebidas proteícas, una a base de quinua malteada y la otra a base de quinua sin maltear (Chenopodium quinoa). Ciencias Agropecuarias, 10.

Casas, N., Salgado, Y., Moncayo, D., \& Cote, S. (2016). Efecto del proceso de malteado en la calidad y estabilidad de una bebida de quinua (Chenopodium quinoa Willd) y mango (Mangifera indica). Agroindustrial Science, Vol. 6(Núm. 1), Pag. 78.

De León, N. (2016). Análisis de las propiedades fisicoquímicas que aporta el maíz negro (Zea Mays L.) en la elaboración de cerveza a tres diferentes temperaturas de fermentación. Universidad de San Carlos de Guatemala, 42.

Drapala, A., \& Hernández, D. (2018). Elaboración de cerveza de maíz (tesis de pregrado). Universidad Nacional de Cuyo.

Faltermaier, A., Waters, D., Becker, T., Arendt, E., \& Gastl, M. (2013). Protein modifications and metabolic changes taking place during the malting of common wheat (triticum aestivum L.). American Society of Brewing Chemists, 153.

Galecio, G., \& Haro, C. (2012). Bebidas fermentadas en base a "Maiz negro" Zea Mays L. Poaceae; con el eco tipo "Racimo de uva" y la variedad 
"Mishca" de la serranía ecuatoriana (tesis pregrado). Universidad Politécnica Salesiana, 84-86.

Harding, M., \& Lovenheim, M. (2014). The Effect of Prices on Nutrition: Comparing the Impact of Product- and Nutrient-Specific Taxes. Journal of Health Economics, 1.

INEN 2304. (2017). Refrescos o bebidas no carbonatadas. Obtenido de http://181.112.149.204/buzon /normas/nte_inen_2304-1.pdf

INEN 2337. (2008). Jugos, pulpas, concentrados, néctares, bebidas de frutas y vegetales. Requisitos. Recuperado el 03 de Enero de 2020, de https://archive.org/stream/ec. nte.2337.2008\#page/n1/mod e/2up

Kok, Y., Ye, L., Muller, J., \& Ow, D. (2018). Kok, Y. J., Ye, L., Muller, J., Ow, D. S.-W., \& Bi, $X$. (2018). Brewing with malted barley or raw barley: what makes the difference in the processes? Applied Microbiology and Biotechnology, 2-3.

Lozano, J. (2006). Color, turbidez, and other sensorial and estructural properties of fruits and fruit products. En J. Lozano, Fruit Manufacturing (pág. 102). Boston: Springer.
Mosher, M., \& Trantham, K. (2016). The "Food" for the Brew. En M. Mosher, \& K. Trantham, Brewing Science: A Multidisciplinary Approach (págs. 132-135). Suiza: Springer.

Olivares, Y., \& Ricaldi, K. (2013). Efecto del proceso del malteado en las características fisicoquímicas y químicas en la obtención de harina de maíz (Zea Mays) (tesis de pregrado). Universidad Nacional del Centro de Perú.

Recalde, M. (2017). Obtencion de una bebida tipo cerveza a partir de maltas de maíz (Zea Mays) y quinua (Chenopodium quinoa). Escuela Politécnica Nacional, 25-26.

Yao, Y., Zhang, R., Wang, B., \& Zhang, S. (2017). Modeling and optimization of anaerobic digestion of corn stover on biogas production: Initial $\mathrm{pH}$ and carbon to nitrogen ratio . Energy Sources, Part A: Recovery, Utilization, and Environmental Effects, 3-4. 